\title{
Vorwort zum 3. Band
}

Das erste Heft des Bayerischen Wörterbuchs erschien im Jahr 1995, der erste Band wurde 2002, der zweite 2012 fertiggestellt. Mit Heft 27 im Jahr 2019 schließt Band 3. Heft 18, der ersten Lieferung von Band 3, ist eine Reihe von Ergänzungen und Berichtigungen zum Quellenheft beigegeben.

Die Redaktion trauert um Klaus Strunk (†2018), der drei Jahrzehnte lang hochgeachteter Vorsitzender der „Kommission für Mundartforschung“" war. Ferner hat die Kommission seit dem Erscheinen von Band 1 ihre Mitglieder Hans Fromm $(† 2008)$ und Johanna Narten $(† 2019)$ durch Tod verloren. Als neue Mitglieder konnten Stephan Elspaß (Salzburg), Hans-Werner Eroms und Rüdiger Harnisch (beide Passau), Mechthild Habermann (Erlangen) und Werner König (Augsburg) hinzugewonnen werden. In der Redaktion fanden ebenfalls Veränderungen statt. Hans Ulrich Schmid wechselte an die Universität Leipzig; Josef Denz und Bernd Insam schieden aus Altersgründen aus - Josef Denz unterstützt die Redaktion weiterhin. Neu hinzugekommen sind Edith Funk (*1956 Krumbach, Initiale E.F.), Andrea Schamberger-Hirt (*1973 Fürstenfeldbruck, Initiale A.S.H.), Michael Schnabel (*1963 Bayreuth, Initiale M.S.) und Felicitas Maria Erhard (*1992 Augsburg, Initiale F.M.E.). Einige Artikel der Strecke PROS-, BRUT- und PS- verfaßte Alexander Glück (*1979 Freising, Initiale A.G.). Die Leitung blieb unverändert bei Anthony Rowley. Im Jahr 2015 hat die Bayerische Akademie der Wissenschaften die Aufsichtsgremien für ihre Forschungsprojekte neu strukturiert. Darüber wird im Vorwort zu Band 4 berichtet.

Grundlegende Änderungen am Konzept und am Erscheinungsbild des Bayerischen Wörterbuchs werden im dritten Band nicht vorgenommen. Von Heft 21 (Sp. 565) an werden Anregungen einer Evaluation des Wörterbuchs umgesetzt. Innerhalb der Wortartikel finden sich feste Fügungen, Vergleiche, Redensarten und Sprichwörter nun unter dem Stichwort „Phraseologie“ (Abkürzung „Phras.") zusammengefaßt.Am Schluß eines Artikels werden nur das „Bayerische Wörterbuch" von Johann Andreas Schmeller und andere ältere Wörterbücher des Bairischen sowie das „Wörterbuch der bairischen Mundarten in Österreich" angeführt; Verweise auf weitere großlandschaftliche Dialektwörterbücher, auf historische Wörterbücher des Deutschen und auf kleinere Dialektwörterbücher entfallen, ebenso die Angabe der Fragebögen, in denen nach dem behandelten Wort explizit gefragt wurde.

Es ist eine angenehme Pflicht, der Stiftung zur Förderung der Wissenschaften in Bayern und der Gesellschaft der Freunde der Bayerischen Akademie der Wissenschaften für die großzügige Unterstützung von Digitalisierungsarbeiten zu danken.

München,im August 2019. 